\title{
Nonadiabatic transitions between lambda-doubling states in the capture of a diatomic molecule by an ion
}

\author{
M. Auzinsh, ${ }^{1}$ E. I. Dashevskaya, ${ }^{2,3}$ I. Litvin, ${ }^{2,4}$ E. E. Nikitin, ${ }^{2,3}$ and J. Troe ${ }^{3,4, a)}$ \\ ${ }^{1}$ Department of Physics, University of Latvia, Riga LV-1586, Latvia \\ ${ }^{2}$ Schulich Faculty of Chemistry, Technion-Israel Institute of Technology Haifa, 32000, Israel \\ ${ }^{3}$ Max-Planck-Institut für Biophysikalische Chemie, Am Fassberg, Göttingen D-37077, Germany \\ ${ }^{4}$ Institut für Physikalische Chemie, Universität Göttingen, Tammannstrasse 6, Göttingen D-37077, Germany
}

(Received 21 February 2008; accepted 2 April 2008; published online 8 May 2008)

\begin{abstract}
The low-energy capture of a dipolar diatomic molecule in an adiabatically isolated electronic state with a good quantum number $\Omega$ (Hund's coupling case $a$ ) by an ion occurs adiabatically with respect to rotational transitions of the diatom. However, the capture dynamics may be nonadiabatic with respect to transitions between the pair of the $\Lambda$-doubling states belonging to the same value of the intrinsic angular momentum $j$. In this work, nonadiabatic transition probabilities are calculated which define the $\Lambda$-doubling $j$-specific capture rate coefficients. It is shown that the transition from linear to quadratic Stark effect in the ion-dipole interaction, which damps the $T^{-1 / 2}$ divergence of the capture rate coefficient calculated with vanishing $\Lambda$-doubling splitting, occurs in the adiabatic regime with respect to transitions between $\Lambda$-doubling adiabatic channel potentials. This allows one to suggest simple analytical expressions for the rate coefficients in the temperature range which covers the region between the sudden and the adiabatic limits with respect to the $\Lambda$-doubling states. (C) 2008 American Institute of Physics. [DOI: 10.1063/1.2913519]
\end{abstract}

\section{INTRODUCTION}

Improving insight into the chemistry of the interstellar medium $^{1}$ has directed attention to the rates of elementary chemical reaction at very low temperatures, among which ion-molecule reactions play an important role (for recent reviews, see, e.g., Refs. 2-5). Under low temperature conditions, capture-controlled processes are most relevant such that capture theories of reaction rates are of particular interest. Such theories are relatively well understood for encounters of ions with electronically closed shell molecules (see, e.g., Refs. 6-8), while capture of open shell species by ions is characterized by a series of complications. Early treatments ${ }^{6,9,10}$ left the impression that the rates diverged at $T \rightarrow 0 \mathrm{~K}$ which, however, clearly is not true. The present series of articles ${ }^{11}$ try to clarify the situation by providing a more elaborate treatment for rates of capture of polar open shell radicals by ions. Our interest not only focuses on temperatures in the $10 \mathrm{~K}$ range of interstellar molecular clouds but we also try to extend the range essentially down to $0 \mathrm{~K} \cdot{ }^{12-14}$ In addition, we try to provide rates for fully stateresolved species.

Following our concept, we have to inspect the basics of scattering theory. State-specific rate coefficients for capture then have to be calculated by solving the scattering equations for a half-collision problem with absorbing boundary conditions at the complex surface, the so-called statistical-closedcoupled method. ${ }^{15-18}$ This general approach is simplified when the capture occurs under adiabatic conditions with respect to transitions between different rotational states. Then, the optimal way to formulate the scattering equations is to

${ }^{a)}$ Electronic mail: shoff@gwdg.de. use the adiabatic rotronic basis since the coupling to adiabatically closed channels, which is present in an arbitrary basis, appears through the adiabatic potentials. This approach, known under different acronyms (statistical adiabatic channel method, ${ }^{19}$ perturbed rotational state method, ${ }^{20,21}$ adiabatic invariance method, ${ }^{22,23}$ adiabatic capture centrifugal sudden approximation ${ }^{24}$ ), uses a classical description for the relative motion of the colliding partners. At very low temperatures, the quantal effects of relative motion begin to show up, and the transition from classical to quantum behavior was elaborated, e.g., in Refs. 12-14. The situation becomes more complicated when open electronic states are involved. The given approaches implicitly assume that the capture occurs in the sudden regime with respect to closely spaced molecular states (spacing much smaller than the spacing between the rotational levels). Such closely spaced molecular states arise as the result of intramolecular nonadiabatic interaction (the $\Lambda$-doubling effect for molecules in an open electronic state) and the hyperfine interaction. If the splitting between such closely spaced states is ignored, the rate coefficient for capture of a dipole molecule in an open electronic state by an ion shows the $T^{-1 / 2}$ divergence in the limit $T \rightarrow 0$. This divergence is an artifact due to assumption of a persistence of the linear Stark interaction ${ }^{6,9,10}$ between a molecule and an ion up to very large separations. In reality, however, the Stark effect becomes quadratic. This removes the intramolecular degeneracy of the rotational states and results in a suppression of the $T^{-1 / 2}$ divergence of the capture rate at very low temperatures. The change in the capture dynamics of a dipolar molecule by an ion with decreasing collision energy (or translational temperature) was qualitatively discussed in Ref. 25. There was, however, no systematic quantitative study of this change because of the compli- 
cated pattern of adiabatic channel potentials and possible nonadiabatic transitions between closely spaced adiabatic channel (AC) states that account for $\Lambda$-doubling and hyperfine interaction. In order to avoid the involved computations of AC potentials with simultaneous $\Lambda$-doubling and hyperfine structure effects and the resulting multichannel nonadiabatic interactions, in the present study, we restrict ourselves to the case that the $\Lambda$-doubling splitting exceeds noticeably the hyperfine structure splitting. Then, it is possible to simplify the general treatment by totally ignoring the hyperfine structure. The effect of the latter is considered in a separate paper devoted to capture of the $\mathrm{NO}$ by $\mathrm{C}^{+}$, see Ref. 26. Our interest in the theory of nonadiabatic transitions between $\Lambda$-doubling levels was also prompted by the possibility to experimentally investigate inelastic scattering from individual $\Lambda$-doubling states, for instance, in collisions of $\mathrm{NO}\left(X^{2} \Pi_{1 / 2}, j=1 / 2\right)$ and $\mathrm{OH}\left(X^{2} \Pi_{3 / 2}, j=3 / 2\right)$ with noblegas atoms. ${ }^{27-30}$

The aim of this paper is to extend the general adiabatic channel treatment to the case when the capture dynamics with respect to $\Lambda$-doubling states in ion-molecule collisions is essentially nonadiabatic and to illustrate the transition between the sudden and adiabatic regimes with respect to these states. We assume that the molecular state is characterized by Hund's coupling case $a$ (isolated electronic state with the good quantum number $\Omega$, the absolute value of the projection of the electronic angular momentum onto the molecular axis) and completely neglect the hyperfine interaction. In Sec. II, we discuss the hierarchy of approximations in the capture dynamics of a dipolar diatom by an ion. Section III describes the calculation of nonadiabatic transition probabilities between $\Lambda$-doubling adiabatic channel states. Section IV presents calculations of nonadiabatic capture rates under the condition that the $\Lambda$-doubling spacing is much lower than the thermal energy. Section $\mathrm{V}$ considers the adiabatic capture for arbitrary relations between the $\Lambda$-doubling spacing and the thermal energy. Section VI contains a general discussion and our conclusion.

\section{HIERARCHY OF APPROXIMATIONS IN THE CAPTURE DYNAMICS OF A POLAR DIATOM AND AN ION}

$\mathrm{AC}$ states and potentials are generated by diagonalization of the Hamiltonian of the collision partners at a fixed interfragment distance $R$ and by choosing the collision axis $\mathbf{R}$, accounting for the quantization of the intrinsic angular momentum $\mathbf{j}$ of the diatom. For a nonvibrating diatom (or a diatom in a given vibrational state), the Hamiltonian consists of the rotational energy of the diatom and the interaction energy between the diatom and the ion.

The rotational energy spectrum of the diatomic molecule in an open shell degenerate electronic state with a good quantum number $\Omega$, which consists of doublets belonging to the same value of $j$ (the $\Lambda$-doubling phenomenon). These components, conventionally called $e$ and $f$ components, ${ }^{31}$ can also be labeled by the parity index $\varepsilon$ which is related to the parity $\pi$ as $\pi=\varepsilon(-1)^{j-1 / 2}$, see Refs. 32-34. The parity quantum number $\pi$ for the lowest components usually alter- nates along the ladder of the quantum numbers $j$, while $\varepsilon$ remains the same. In this work, we label the lower and upper components by $\varepsilon=+1$ and $\varepsilon=-1$, respectively, in accord with the assignment for $\mathrm{NO}\left(X^{2} \Pi_{1 / 2}\right)$, see Ref. 34. The rotational energy levels then are written as $E_{j, \varepsilon}$, and the splitting $\Delta E_{j}=E_{j,-1}-E_{j,+1}$ between the components of the doublet results from the rotational nonadiabatic and spin-orbit coupling of the given electronic state to other electronic states.

The interaction between the diatom and the ion is taken in a form appropriate for low-energy collisions, i.e., it includes only the long-range limit of the ion-molecule interaction. Namely, it includes the charge-permanent dipole, charge-permanent quadrupole, and charge-induced dipole terms. ${ }^{35}$ For simplicity, we neglect the anisotropy in the charge-induced dipole term and assume that the quadrupole tensor is axially symmetric. In this way, we consider an interaction potential of the form

$$
V(R, \gamma)=\frac{q \mu_{D}}{R^{2}} P_{1}(\cos \gamma)+\frac{q Q}{R^{3}} P_{2}(\cos \gamma)-\frac{q^{2} \alpha}{2 R^{4}},
$$

where $\gamma$ is the angle between the collision axis $\mathbf{R}$ (directed from the center of mass of the diatom toward the ion) and the molecular axis $\mathbf{r}$ (directed along the dipole moment vector), $P_{1}$ and $P_{2}$ are the Legendre polynomials, $\mu_{D}, Q$, and $\alpha$ are the dipole moment, quadrupole moment, and the mean polarizability of the diatom, respectively, and $q$ is the charge of the ion.

If the Hamiltonian $\hat{H}$ is diagonalized at a fixed interfragment distance $R$ in the set of the free-molecule functions $|\Omega, \varepsilon, j, \widetilde{\omega}|^{\text {free }}$, with $\widetilde{\omega}$ being the projection of the intrinsic angular momentum onto the space-fixed quantization axis $\mathbf{R}$, it yields, as its eigenvalues, the AC energies $W(R)$. They can be labeled by the exact quantum number $\widetilde{\omega}$ and a set of new quantum numbers (we omit from now on the quantum number $\Omega$ which is the same for all states). These new quantum numbers can be written as $j, \varepsilon$, understanding that they have their usual meaning only asymptotically (at the limit $R \rightarrow \infty$ ) such that they are established by the adiabatic correlation of $W_{j, \varepsilon, \tilde{\omega}}(R)$ with $E_{j, \varepsilon}$. Once the eigenvalues $W_{j, \varepsilon, \tilde{\omega}}(R)$ are written as

$$
W_{j, \varepsilon, \widetilde{\omega}}(R)=E_{j, \varepsilon}+\widetilde{V}_{j, \varepsilon, \widetilde{\omega}}(R),
$$

they define the AC potentials $\widetilde{V}_{j, \varepsilon, \tilde{\omega}}(R)$. Note that the AC states $|\varepsilon, j, \widetilde{\omega}\rangle^{\mathrm{AC}}$ are doubly degenerate with respect to the sign of $\widetilde{\omega}$ such that the AC potentials can be written as $\tilde{V}_{j, \varepsilon, \omega}(R)$, where $\omega=|\widetilde{\omega}|$.

Calculations of state-specific cross sections and capture rate coefficients, in principle, should use AC potentials $\widetilde{V}_{j, \varepsilon, \omega}(R)$. However, the general treatment can be simplified under conditions when nonadiabatic transitions between certain groups of states fall into the sudden or adiabatic regimes. In the sudden regime, the population of the respective AC states becomes statistical before the system reaches the characteristic distance which corresponds to capture. Therefore, the specification of the initial states with the quantum numbers within this group is redundant, and these states can be removed both from the $\mathrm{AC}$ potentials and the scattering equations. A well-known example of this approach is the 


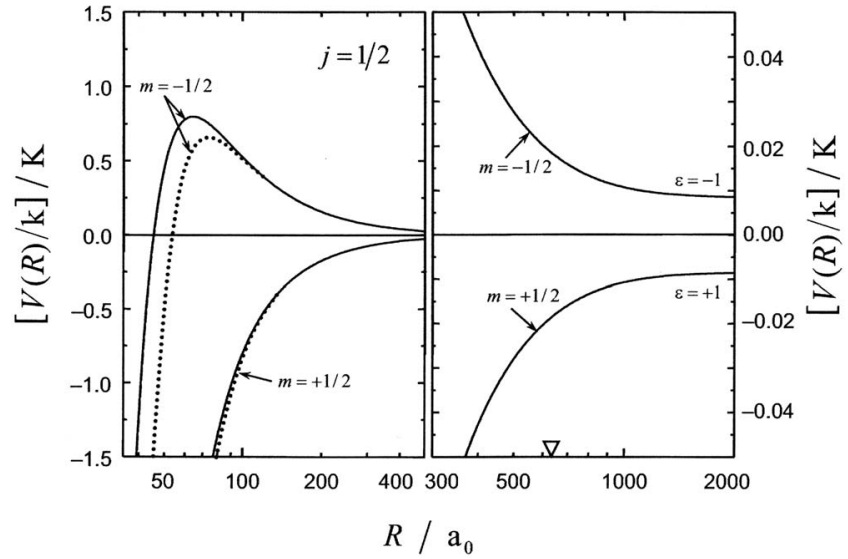

FIG. 1. AC potentials for capture of $\mathrm{NO}\left(X^{2} \Pi_{1 / 2}, j=1 / 2\right)$ by a positive ion relative to the mean rotational energy of $\varepsilon=1$ and $\varepsilon=-1$ states. The AC PR potentials are shown by the full lines, accurate AC potentials by dotted lines (see text for details). The triangle marks the distance $R_{1 / 2,1 / 2}^{(r)}=624$ a.u.

calculation of rotationally specific capture rate coefficients $k_{j}$, which implicitly assumes that the capture is sudden with respect to transitions between $\Lambda$-doubling components. Then AC potentials are calculated from the Hamiltonian with the rotational energy of the diatom without accounting for the $\Lambda$-doubling. In our work, ${ }^{11}$ these AC potentials for a molecule in an isolated state of Hund's coupling case $a$ with the quantum number $\Omega$ were written as $V_{j, m}^{\text {Acc }}(R)$. The quantum number $m$ was defined through its absolute value $|m|=\omega$ and the condition that the positive/negative values of $m$ correspond to the attractive/repulsive charge-permanent dipole interaction. (This definition coincides with the alternative definition $m=-|\widetilde{\omega}| \operatorname{sign}(\widetilde{\Omega} \widetilde{\omega})$, where $\widetilde{\Omega}$ is the projection of the intrinsic angular momentum onto the molecular axis with the convention that the direction of the molecular axis coincides with that of the dipole moment and the molecule interacts with a positive ion.) For the sake of simplicity, we will write $V_{j, m}^{\mathrm{Acc}}(R)$ simply as $V_{j, m}(R)$. At large distances, AC potentials can be calculated analytically in the so-called perturbed rotor (PR) approach which retains the same powers of $R$ as the potential in Eq. (1). It incorporates first-order chargepermanent dipole (cd) (proportional to $R^{-2}$ ), first-order charge-permanent quadrupole (cq) (proportional to $R^{-3}$ ), and the Langevin $(L)$ term consisting of the charge-induced dipole (cid) plus second-order charge-permanent dipole terms (both proportional to $R^{-4}$ ). The general expression for $V_{j, m}^{\mathrm{PR}}(R)$ for an isolated electronic state $|\Omega\rangle$ reads as ${ }^{11}$

$$
V_{j, m}^{\mathrm{PR}}(R)=V_{j, m}^{\mathrm{cd}}(R)+V_{j, m}^{\mathrm{cq}}(R)+V_{j, m}^{L}(R),
$$

with the following partial contributions:

$$
\begin{aligned}
& V_{j, m}^{\mathrm{cd}}(R)=-s_{m} a_{j, m} / R^{2}, \\
& a_{j, m}=q \mu_{D}|m| \Omega / j(j+1), \\
& V_{j, m}^{\mathrm{cq}}(R)=b_{j, m} / R^{3}, \\
& b_{j, m}=\frac{q Q\left(j(j+1)-3 m^{2}\right)}{(2 j+3)(2 j-1)} \frac{\left(j(j+1)-3 \Omega^{2}\right)}{j(j+1)},
\end{aligned}
$$

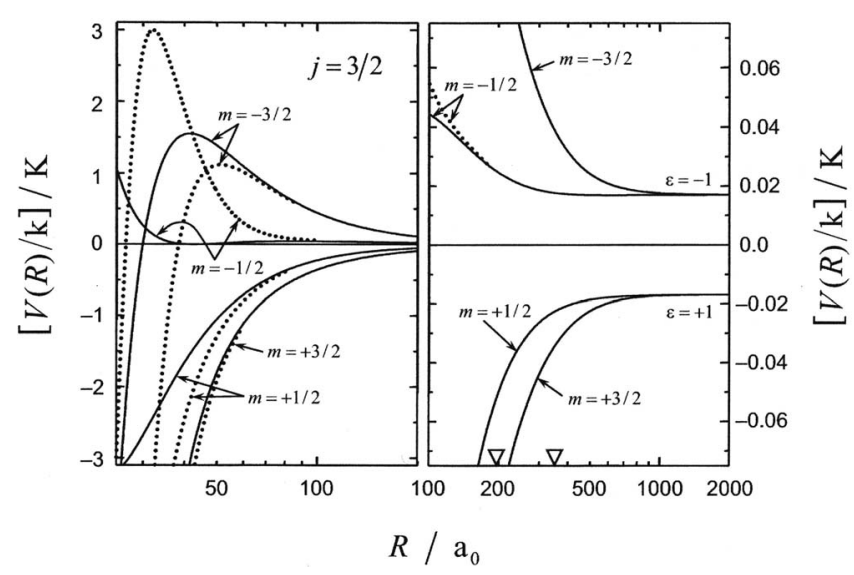

FIG. 2. As in Fig. 1, for $j=3 / 2$. The triangles mark the distances $R_{3 / 2,1 / 2}^{(r)}$ $=197$ a.u. and $R_{3 / 2,3 / 2}^{(r)}=342$ a.u.

$$
\begin{aligned}
V_{j, m}^{L}(R) & =c_{j, m} / R^{4}, \\
c_{j, m}= & -\frac{\alpha q^{2}}{2}+\frac{\mu_{D}^{2} q^{2}}{2 B}\left(\frac{\left(j^{2}-\Omega^{2}\right)\left(j^{2}-m^{2}\right)}{j^{3}(2 j-1)(2 j+1)}\right. \\
& \left.-\frac{\left((j+1)^{2}-\Omega^{2}\right)\left((j+1)^{2}-m^{2}\right)}{(j+1)^{3}(2 j+1)(2 j+3)}\right),
\end{aligned}
$$

where $B$ is the rotational constant of the diatom (in energy units), $s_{m}=\operatorname{sign}(m)$, and $a_{j, m}$ is positive. In Eq. (4), we write $s_{m}|m|$ instead of $m$ in anticipation of a generalization for $\Lambda$-doubling effect [see Eq. (7) below].

If the effects of $\Lambda$-doubling are included, the calculation of the AC potentials, in general, should be performed taking nonadiabatic interactions with higher electronic states into account. However, this complication could not be relevant if the $\Lambda$-doubling splitting is much smaller than the energy for rotational transitions. Then, the effects of $\Lambda$-doubling will show up when the AC potentials $V_{j, m}(R)$ are adequately given by their PR counterparts, $V_{j, m}^{\mathrm{PR}}(R)$. The latter can be modified in such a way that the term, which represents the linear Stark effect, is transformed into a term describing an intermediate Stark effect such that $V_{j, m}^{\mathrm{PR}}(R)$ is changed into $\widetilde{V}_{j, m}^{\mathrm{PR}}(R)$. This transformation can be easily accomplished because, first, the quantum number $j$ in the PR region can be

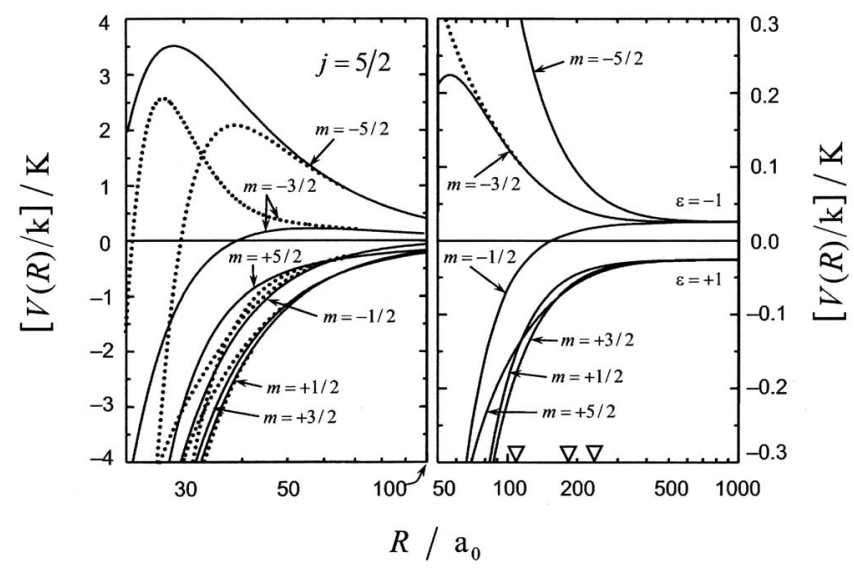

FIG. 3. As in Fig. 1, for $j=5 / 2$, The triangles mark the distances $R_{5 / 2,1 / 2}^{(r)}$ $=106$ a.u., $R_{5 / 2,3 / 2}^{(r)}=183$ a.u., and $R_{5 / 2,5 / 2}^{(r)}=236$ a.u. 
identified to the rotational quantum number of the diatom, and, second, the wave functions of the $\Lambda$-components of the diatom with the quantum number $j$ are positive and negative linear combinations of $\Omega$ and $-\Omega$ states. ${ }^{31}$ Since only the charge-dipole interaction mixes free states of different parity, the $\Lambda$-doubling effect modifies solely the cd term in the PR potential. Namely, the term $\tilde{V}_{j, m}^{\text {cd }}$, which describes the intermediate Stark effect for cd interaction and includes the $\Lambda$-doubling splitting $\Delta E_{j}$, when presented in the $j, m$ nomenclature, reads as

$$
\tilde{V}_{j, m}^{\mathrm{cd}}(R)=-\frac{s_{m}}{2} \sqrt{\left(\Delta E_{j}\right)^{2}+\left(2 a_{j, m} / R^{2}\right)^{2}}+s_{m} \Delta E_{j} / 2,
$$

where again positive/negative values of $m$ refer to the attractive/repulsive cd interaction in the intermediate Stark effect regime. In the limit $R \rightarrow \infty$, because of the adiabatic correlation $\varepsilon=\operatorname{sign}(m), \tilde{V}_{j, m}^{\mathrm{cd}}(R)$ converges to $E_{j, \varepsilon}$, and for $\Delta E_{j} \rightarrow 0, \tilde{V}_{j, m}^{\mathrm{cd}}(R)$ passes into $V_{j, m}^{\mathrm{cd}}(R)$. Therefore, the full PR AC potentials that take into account the $\Lambda$-doubling effects can be written as

$$
\tilde{V}_{j, m}^{\mathrm{PR}}(R)=\widetilde{V}_{j, m}^{\mathrm{cd}}(R)+V_{j, m}^{\mathrm{cq}}(R)+V_{j, m}^{L}(R),
$$

which has the following $R$-dependence in the regions of the linear and quadratic Stark effect:

$$
\tilde{V}_{j, m}^{\mathrm{PR}}(R) \rightarrow \begin{cases}s_{m} a_{j, m} / R^{-2}+b_{j, m} R^{-3}+c_{j, m} R^{-4}=V_{j, m}^{\mathrm{PR}}(R) & \text { for } a_{j, m} R^{-2} \gg \Delta E_{j} \\ b_{j, m} R^{-3}+\left(c_{j, m}-s_{m} a_{j, m}^{2} / \Delta E_{j}\right) R^{-4} & \text { for } a_{j, m} R^{-2} \ll \Delta E_{j},\end{cases}
$$

The given hierarchy of the AC potentials will be used in our calculation of state-specific capture rate coefficients for different temperature ranges specified by the characteristic temperature $T^{\Lambda}$ for $\Lambda$-doubling $\left(T^{\Lambda} \approx \Delta E_{j} / k_{B}\right)$.

Finally, we reiterate that an $\mathrm{AC}$ state with the quantum number $m$ is doubly degenerate with respect to the two possible signs of $\widetilde{\omega}= \pm|m|$ or to the two possible values of the symmetry index $\sigma$ related to the reflection of the AC state at a plane passing through the collision axis. This degeneracy is lifted when one passes from the $\mathrm{AC}$ approximation to the axially nonadiabatic channel (ANC) approach when each ANC state will correspond to a definite value of the total angular momentum and a total parity of the collision complex.

As an illustration to the above, we show AC potentials for the system of a positive ion $+\mathrm{NO}\left(X^{2} \Pi_{1 / 2}, j, \varepsilon\right)$ with $j$ $=1 / 2$ (Fig. 1), $j=3 / 2$ (Fig. 2), and $j=5 / 2$ (Fig. 3). In these figures, the AC PR potentials $\widetilde{V}_{j, m}^{\mathrm{PR}}(R)$ are shown by full lines and accurate potentials (shown in the regions where they noticeably deviate from the PR potentials) by the dotted lines. The energy range in the right part of these figures is chosen such that it illustrates the potentials in the region of the intermediate Stark interaction; here, the potentials are given relative to the mean rotational energy level, with a $\Lambda$-doubling splitting which increases linearly with $j$ $\left[\Delta E_{j} / k_{B}=0.017(j+1 / 2) \mathrm{K}\right]$, see Ref. 36. The energy range in the left parts of these figures is chosen such that it shows the potential barriers for the upper AC potentials. In this range, the potentials $\tilde{V}_{j, m}$ are well approximated by $V_{j, m}$, implying that the $\Lambda$-splitting effect can be neglected. The left and right parts cover a common range of $\mathrm{NO}$-ion distances to facilitate the comparison of the potentials shown for different energy ranges. The following comments to Figs. 1-3 are in order. (i) Fig. 1, $j=1 / 2$. The symmetry of the AC potentials with respect to the zero line in the right part of the figure indicates the absence of a first-order chargequadrupole interaction and an only negligible contribution of the charge-induced dipole interaction. The AC PR potentials in the left part qualitatively correctly predict the potential barrier. As shown in Ref. 11, the AC PR potentials can be used for the approximate calculation of the reduced rate coefficient for capture of $\mathrm{NO}\left(X^{2} \Pi_{1 / 2}, j=1 / 2\right)$ by $\mathrm{C}^{+}$at $T>0.1 \mathrm{~K}$, also see below.

(ii) Fig. 2, $j=3 / 2$. The slight asymmetry of the AC potentials with respect to the zero line in the right part of the figure indicates a noticeable effect of the chargequadrupole interaction and some contribution of the charge-induced dipole interaction. One also sees an incipient deviation of the accurate potential with $m$ $=1 / 2$ from its AC counterpart. The accurate potentials in the left part noticeably deviate from their AC PR counterparts. The attractive interaction is weaker compared to the $j=1 / 2$ case which is due to the result of the smaller projection of the molecular dipole moment onto the collision axis. As a result, the capture rate coefficient for $j=3 / 2$ is noticeably smaller than that for $j=1 / 2$, see Ref. 11 .

(iii) Fig. 3, $j=5 / 2$. The strong asymmetry of the AC potentials with respect to the zero line in the right part of the figure indicates a larger effect of the chargequadrupole interaction and a noticeable contribution of the charge-induced dipole interaction. A new feature (compared to $j=1 / 2$ and $j=3 / 2$ ) is the attractive AC potential with $m=1 / 2$ that correlates with the upper $\Lambda$-component of the free molecular state. We note in passing that the large- $R$ representation of 
$\tilde{V}_{j, m}^{\mathrm{PR}}$ [charge-quadrupole plus modified Langevin interaction, see Eq. (9)] provides a good approximation to $\widetilde{V}_{j, m}^{\mathrm{PR}}$ for $R>300$ a.u., where the interaction becomes smaller that about $0.02 \mathrm{~K}$. The accurate potentials in the left part quite strongly deviate from their AC PR counterparts. The rate coefficient for $j=5 / 2$ is slightly smaller than that for $j=3 / 2$, and its temperature dependence shows features which are similar to the rate coefficient for capture of a molecule with vanishing dipole moment. ${ }^{11}$

Also shown in Figs. 1-3 (by triangles on the abscissa) are the locations $R_{j, m}^{(r)}$ of possible nonadiabatic transitions between the AC states $|j, m\rangle$ and $|j,-m\rangle$ (see next section).

\section{NONADIABATIC TRANSITIONS FOR $\Lambda$-DOUBLING STATES}

For temperatures $T \gg T^{\Lambda}$, the passage of the potential barriers by the collision partners toward the complex boundary occurs under conditions where the effective AC potentials between the molecule and the ion are governed by linear Stark effect. However, during their mutual approach to the barriers, the partners pass a region where the quadratic Stark effect changes into linear Stark effect, and in this region nonadiabatic transitions between the AC potentials $\widetilde{V}_{j, m}^{\mathrm{PR}}$ and $\tilde{V}_{j,-m}^{\mathrm{PR}}$ are possible. The nonadiabatic transition probability $P_{j, m}$ should be found from the solution of two coupled equations containing the amplitudes of the $|j, m\rangle\rangle^{\mathrm{AC}}$ and $|j,-m\rangle^{\mathrm{AC}}$ states. Under the condition when the $\Lambda$-doubling spacing $\Delta E_{j}$ is much smaller than the collision energy $E$, these equations can be formulated in the "impact parameter approximation" (i.e., in the common trajectory approximation for a rectilinear trajectory with a fixed velocity $\nu$ ) of relative motion. The most important quantity that determines the nonadiabatic transition probability is the Massey parameter $\xi_{j, m}$. According to the standard procedure, ${ }^{37}$ it is defined through the energy spacing $\Delta E_{j}$, collision velocity $\nu$, and the distance $R_{j, m}^{(i)}$ from the value $R_{j, m}^{c}$ at which the AC potentials $\tilde{V}_{j, m}^{\mathrm{PR}}$ and $\widetilde{V}_{j,-m}^{\mathrm{PR}}$ cross. Implicitly, $R_{j, m}^{(i)}=\operatorname{Im}\left\{R_{j, m}^{c}\right\}$, where $R_{j, m}^{c}$ is found from the equation $\widetilde{V}_{j, m}^{\mathrm{PR}}\left(R_{j, m}^{c}\right)=\widetilde{V}_{j,-m}^{\mathrm{PR}}\left(R_{j, m}^{c}\right)$. Yet another characteristics of the nonadiabatic event is $R_{j, m}^{(r)}=\operatorname{Re}\left\{R_{j, m}^{c}\right\}$, which approximately gives the position of the region where nonadiabatic transitions occur. Taking the potentials in the form of Eqs. (3) and (4), we find $R_{j, m}^{c}$ $=\sqrt{2 a_{j m} / \Delta E_{j}} \exp (i \pi / 4)$, i.e., $R_{j, m}^{(i)}=R_{j, m}^{(r)}=\sqrt{a_{j m} / \Delta E_{j}}$. The distances $R_{j, m}^{(r)}$ for the NO+ion system are shown in Figs. 1-3.

The Massey parameter for nonadiabatic transitions between the AC states $|j, m\rangle$ and $|j,-m\rangle$ is written as

$$
\xi_{j, m}=\frac{\Delta E_{j} R_{j, m}^{(i)}}{\hbar v}=\frac{1}{2} \sqrt{\frac{\Delta E_{j}}{E} \frac{2 \mu a_{j, m}}{\hbar^{2}}},
$$

where $\mu$ is the reduced mass of the colliding partners. If it is large, the dimensionless quantity $\sqrt{2 \mu a_{j, m} / \hbar^{2}}$ can be interpreted as the classical counterpart $\widetilde{J}_{j, m}$ of the quantum number of total angular momentum for which the centrifugal repulsion $\left(\hbar \widetilde{J}_{j, m}\right)^{2} / 2 \mu R^{2}$ compensates the first-order chargedipole attraction, $-a_{j, m} / R^{2}$. Unless $a_{j, m}$ is pathologically small, $\widetilde{J}_{j, m}$ is a large number. ${ }^{11}$ Thus, Eq. (10) can be rewritten as the product of small and large factors

$$
\xi_{j, m}=\frac{1}{2} \sqrt{\frac{\Delta E_{j}}{E}} \widetilde{J}_{j, m}
$$

such that $\xi_{j, m}$ may be of the order of unity even for a small value of the ratio $\Delta E_{j} / E$. We therefore conclude that, for $T$ $>T^{\Lambda}$, the capture may occur in the nonadiabatic regime with respect to transitions between the $\Lambda$-doubling components of a given rotational state. With decreasing temperature, the ratio $\Delta E_{j} / E$ increases, and the capture dynamics, still with $\Delta E_{j} / E \ll 1$, becomes adiabatic (i.e., $P_{j, m}$ becomes negligibly small).

The nonadiabatic dynamics in the passage from the region of the quadratic Stark effect to the linear Stark effect, under the condition $\Delta E_{j} / E \ll 1$, is described by coupled timedependent equations for the amplitudes of the AC states evolving along a straight-line trajectory. One can use different basis functions in writing these equations. Here, we adopt the basis of the AC states without the $\Lambda$-doubling interaction. In this representation, the charge-dipole interaction is diagonal, while the $\Lambda$-doubling interaction appears as a coupling.

Omitting the subscripts in $a$ and $\Delta E$, the equations for the amplitudes $A_{+}$and $A_{-}$of these basis states are written in the form

$$
\begin{aligned}
& i \hbar \frac{d}{d t} A_{-}=+\frac{a}{R^{2}} A_{-}+\frac{\Delta E}{2} A_{+}, \\
& i \hbar \frac{d}{d t} A_{+}=-\frac{a}{R^{2}} A_{+}+\frac{\Delta E}{2} A_{-} .
\end{aligned}
$$

These equations should be integrated along a trajectory with the impact parameter $b$ such that

$$
R(t)=\sqrt{b^{2}+v^{2} t^{2}} .
$$

If we introduce a scaled distance $\rho=R / R_{0}$, a scaled impact parameter $\beta=b / R_{0}$, and a scaled linear distance $\varsigma=\sqrt{\rho^{2}-\beta^{2}}$ with $R_{0}=\sqrt{a / \Delta E}$, Eq. (12) transforms into

$$
\begin{aligned}
& \frac{i}{\xi} \frac{d}{d \varsigma} A_{-}=+\frac{1}{\varsigma^{2}+\beta^{2}} A_{-}+\frac{1}{2} A_{+}, \\
& \frac{i}{\xi} \frac{d}{d \varsigma} A_{+}=-\frac{1}{\varsigma^{2}+\beta^{2}} A_{+}+\frac{1}{2} A_{-},
\end{aligned}
$$

where $\xi=\Delta E R_{0} / \hbar \nu$.

For the calculation of the transition probability for a single passage of the coupling region, Eq. (14) should be integrated from small $\rho$ where $1 / \rho^{2} \gg 1$ (region of the linear Stark effect) to large $\rho$ where $1 / \rho^{2} \ll 1$ (region of the quadratic Stark effect), with the initial conditions

$$
\left.A_{-}(\rho)\right|_{\text {small } \rho}=1,\left.\quad A_{+}(\rho)\right|_{\text {small } \rho}=0 .
$$

Then, the transition probability is equal to

$$
P=(1 / 2)\left|A_{+}(\rho)\right|_{\text {large } \rho}-\left.\left.A_{-}(\rho)\right|_{\text {large } \rho}\right|^{2} .
$$

In this way, the transition probability $P$ will depend on the two parameters $\xi$ and $\beta$, i.e., $P=P(\xi, \beta)$. The transition prob- 


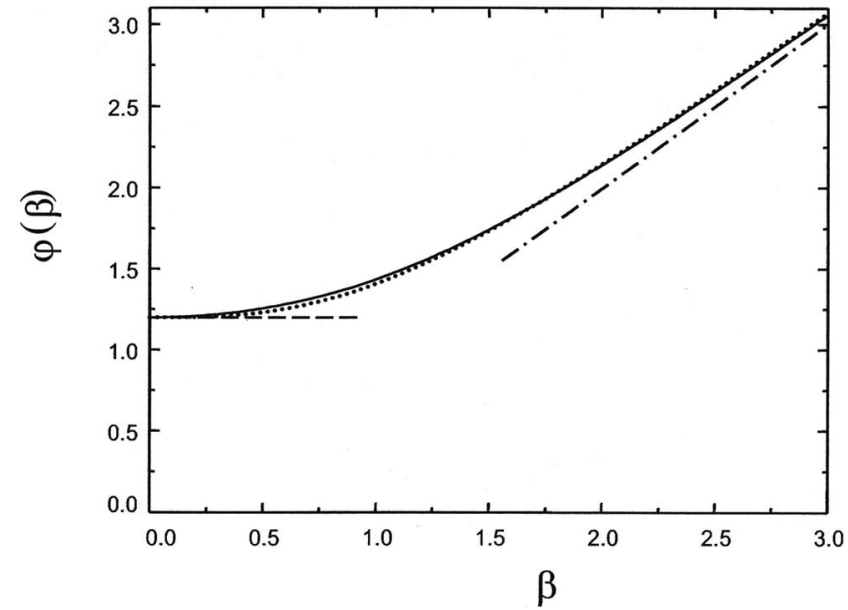

FIG. 4. Dependence of the factor $\varphi(\beta)$ in Eq. (18) on the reduced impact parameter $\beta$ (dotted curve) and its analytical approximation (full curve). The dashed and dash-dotted lines correspond to the asymptotics of $\varphi(\beta)$ in Eq. (20).

ability $P_{j, m}$ introduced above is recovered from the function $P(\xi, \beta)$ as $P_{j, m}(E, b)=P\left(\xi_{j, m}, \beta_{j, m}\right)$ with $\xi_{j, m}$ defined by Eqs. (10) and (11) and $\beta_{j, m}=b / R_{j, m}^{(r)}$.

Two limiting expressions for $P=P(\xi, \beta)$ are known. In the sudden limit, for $\xi \rightarrow 0$, the transition probability $P$ $=P_{\text {sudd }}$ can be calculated as the square of the projection of the $|j, \varepsilon\rangle$ state onto the $|j, \widetilde{\Omega}\rangle$ state which yields $P_{\text {sudd }}=1 / 2$. In the near-adiabatic limit, for $\xi \gg 1$, when the transition probability is exponentially small, it is given by the standard exponential expression ${ }^{37}$

$$
P_{\text {near adiab }}=\exp \left\{-\frac{2}{\hbar} \operatorname{Im} \int_{t_{r}}^{t_{c}} \sqrt{(\Delta E)^{2}+4 a^{2} / R^{4}(t)} d t\right\},
$$

where $t_{r}$ is an arbitrary real time and $t_{c}$ is a complex time which corresponds to the vanishing value of the square root. When the integral in the exponent is expressed in the reduced parameters, Eq. (17) reads as

$$
\begin{aligned}
& P_{\text {near adiab }}(\xi, \beta)=\exp (-2 \xi \varphi(\beta)), \\
& \varphi(\beta)=\operatorname{Im} \int_{\varsigma_{r}}^{\varsigma_{e}} \sqrt{1+4 /\left(\varsigma^{2}+\beta^{2}\right)^{2}} d \varsigma .
\end{aligned}
$$

The limiting values of $\varphi(\beta)$ are

$$
\varphi(\beta)= \begin{cases}1.198 & \text { for } \beta=0 \\ \beta & \text { for } \beta \gg 1 .\end{cases}
$$

The function $\varphi(\beta)$ is illustrated in Fig. 4. Also shown is a simple analytical approximation

$$
\varphi(\beta) \approx \varphi(0)\left(1+(\beta / \varphi(0))^{n}\right)^{1 / n}
$$

with $n=2.9$.

As noted above, in general, the transition probability depends on the two parameters: $\xi$ and $\beta$. However, the dependence on the reduced impact parameter $\beta$ can be neglected if the probabilities are used for calculations of capture rate coefficients under the condition $\Delta E \ll E$. Indeed, the capture

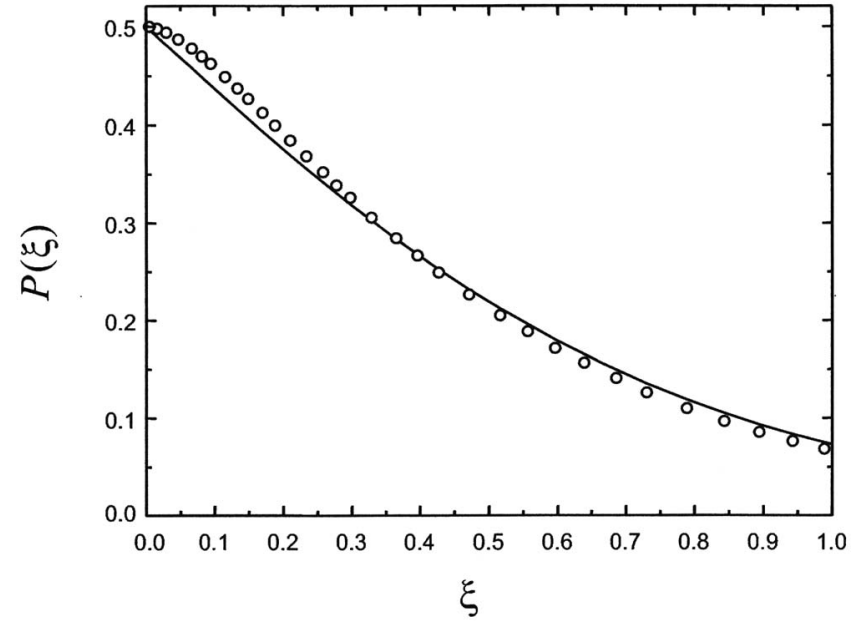

FIG. 5. Nonadiabatic transition probability $P(\xi)$ for intermediate Stark effect adiabatic channels as a function of the Massey parameter $\xi$. The full line corresponds to the analytical approximation by Eq. (21), the symbols are numerically accurate transition probabilities.

distance $R_{c}$ is of the order of $\sqrt{a / E}$, while the characteristic value of the impact parameter, which affects the transition probability, is of the order of $\sqrt{a / \Delta E}$. Since the former is substantially smaller than the latter, we can simplify the transition probability $P$ in the range of small $\beta$, which is of interest for the capture, by writing $\varphi(\beta) \approx \varphi(0)$ (see Fig. 4) such that $P(\xi, \beta) \approx P(\xi, \beta)_{\beta=0} \equiv P(\xi)$. The bridging between the sudden and near-adiabatic limits can be accomplished by using the analogy to the Rozen-Zener-Demkov model, ${ }^{37}$ which gives an analytical expression of the transition probability for arbitrary values of the Massey parameter. In this way, the transition probability $P(\xi)$ between the nearadiabatic and sudden limits can be approximated by

$$
P^{\text {app }}(\xi)=\frac{\exp (-c \xi)}{1+\exp (-c \xi)},
$$

where $c=2 \varphi(0)=2.396$. Figure 5 compares numerically accurate transition probabilities $P(\xi)$ with the approximation in Eq. (21).

\section{RATE COEFFICIENTS FOR $\Lambda$-DOUBLING SPECIFIC CAPTURE IN THE NONADIABATIC REGIME}

We now turn to the rate coefficients for capture in individual $\Lambda$-doublet states. These will be derived by a generalization of our earlier treatment from Ref. 11. The reduced partial and total $\mathrm{AC}$ capture rate coefficients $\chi_{j, m}^{\mathrm{AC}}, \chi_{j}^{\mathrm{AC}}$ (i.e., the ratio of the partial rate coefficients to the Langevin capture rate coefficient $k^{\mathrm{L}}=2 \pi q \sqrt{\alpha / \mu}$ ), which were introduced in Ref. 11, correspond to the sudden transition between the $\Lambda$ components, and are written as

$$
\chi_{j, m}^{\mathrm{AC}}=A(T) \int_{0}^{\infty} \frac{J d J}{2 j+1} \exp \left(-U_{j, m}^{\max }(J) / k_{B} T\right),
$$




$$
\chi_{j}^{\mathrm{AC}}=\sum_{m=-j}^{j} \chi_{j, m}^{\mathrm{AC}},
$$

where $J$ is a classical counterpart of the quantum number of the total angular momentum, and the factor $A(T)$ is represented as

$$
A(T)=\sqrt{\frac{8 k_{B} T}{\pi \mu}} \frac{\pi \hbar^{2}}{\mu k_{B} T} \frac{1}{2 \pi q} \sqrt{\frac{\mu}{\alpha}} .
$$

The energy $U_{j, m}^{\max }(J)$ is defined through the properties of the effective AC potentials, composed of the AC potential and the classical centrifugal energy,

$$
U_{j, m}(R, J)=\frac{\hbar^{2}(J+1 / 2)^{2}}{2 \mu R^{2}}+V_{j, m}(R) .
$$

The quantity $U_{j, m}^{\max }(J)$ in Eq. (22) corresponds to the maximum of the effective potential $U_{j, m}(R, J)$ if the latter possesses a barrier; it is equal to zero if $U_{j, m}(R, J)$ is attractive, and infinity if $U_{j, m}(R, J)$ is repulsive. The asymptotic form of $\chi_{j, m}^{\mathrm{AC}}$ for temperatures substantially lower or higher than the characteristic temperature, $T^{\mathrm{cd}}=\Omega^{2} m^{2} \mu_{D}^{2} / j^{2}(j+1)^{2} \alpha k_{B}$, is obtained from Eqs. (22) and (24) when only cd or cid terms in the AC potential $V_{j, m}(R)$, respectively, are used. One then has

$$
\chi_{j, m}^{\mathrm{AC}}= \begin{cases}\chi_{j, m}^{\mathrm{cd}}=\frac{1}{2 j+1} \sqrt{\frac{2}{\pi \alpha k_{B} T} \frac{\Omega m \mu_{D}}{j(j+1)} \Theta(m),}, \quad T \ll T^{\mathrm{cd}} \\ \chi_{j, m}^{\mathrm{cid}}=\frac{1}{2 j+1}, & T \gg T^{\mathrm{cd}} .\end{cases}
$$

In this expression, $\Theta(m)$ is a step function which selects the partial rate coefficients that correspond to attractive cd interaction. The lack of a dependence of the partial Langevin rate coefficients $\chi_{j, m}^{\text {cid }}$ on $m$ implies that all potentials with attractive and repulsive cd terms lead to capture.

We now generalize Eq. (22) by taking into account the nonadiabatic transitions. The expression for the rate coefficient $\chi_{j, m}^{\mathrm{AC}}$ generates two quantities, namely, $\chi_{j, m}^{\text {trans }}$, which is related to the nonadiabatic transition (trans) and $\chi_{j, m}^{\text {surv }}$, which is related to adiabatic survival (surv). Both quantities require thermal averaging of the transition/survival probabilities. Explicitly, we write

$$
\begin{aligned}
\chi_{j, m}^{\text {surv/trans }}= & A(T) \int_{0}^{\infty} \frac{J d J}{2 j+1} \int_{U_{j, m}^{\max }(j)}^{\infty} P_{j, m}^{\text {surv } / \text { trans }}(E) \\
& \times \exp \left(-E / k_{B} T\right) \frac{d E}{k_{B} T},
\end{aligned}
$$

where the probabilities $P_{j, m}^{\text {surv/trans }}$ are related to $P_{j, m}$ as

$$
\begin{aligned}
& P_{j, m}^{\text {surv }}(E)=1-P_{j, m}(E), \\
& P_{j, m}^{\text {trans }}(E)=P_{j, m}(E) .
\end{aligned}
$$

One should note that $\chi_{j, m}^{\text {trans }}+\chi_{j, m}^{\text {surv }}=\chi_{j, m}^{\mathrm{AC}}$ and that, in the sudden limit, $\chi_{j, m}^{\text {trans }}=\chi_{j, m}^{\text {surv }}=\chi_{j, m}^{\mathrm{AC}} / 2$. The quantities in Eq. (26) de- termine the partial $\tilde{\chi}_{j, m}$ and the total $\tilde{\chi}_{j, \varepsilon}$ rate coefficients from the $j, \varepsilon$ states of the $\Lambda$-doublet,

$$
\begin{aligned}
& \tilde{\chi}_{j, m}=2 \chi_{j, m}^{\text {surv }}+2 \chi_{j,-m}^{\text {trans }}, \\
& \tilde{\chi}_{j, \varepsilon}=\sum_{m} \tilde{\chi}_{j, m},
\end{aligned}
$$

where the allowed values of $m$ are determined by the parity index $\varepsilon$ from the adiabatic correlation $\varepsilon=\operatorname{sign}(m)$. [The factor 2 at the right hand side of Eq. (28) is due to the fact that the normalizations of the rate in this and in the earlier paper ${ }^{11}$ differ; $\tilde{\chi}_{j, m}$ is defined as the rate per unit population of each $\Lambda$ component, while $\chi_{j, m}$ was defined as the rate per unit total population of both $\Lambda$ components]. Equation (28) leads to

$$
\tilde{\chi}_{j, \varepsilon}+\tilde{\chi}_{j,-\varepsilon}=2 \chi_{j}^{\mathrm{AC}} .
$$

One, therefore, can observe a splitting of the rate coefficients, for capture in the rotational state $j$ with $\Lambda$-doubling effect neglected, into two components which correspond to capture in the rotational states $j, \varepsilon=+1$ and $j, \varepsilon=-1$ being separated by the energy spacing $\Delta E_{j}$.

Capture for the $\Lambda$-doublet, with arbitrary populations $n_{j,+}$ and $n_{j,-}$ of its components, can be characterized by an effective rate coefficient $\widetilde{\chi}_{j}^{\text {eff }}$ as

$$
\tilde{\chi}_{j}^{\mathrm{eff}}=\frac{\tilde{\chi}_{j,+} n_{j,+}+\tilde{\chi}_{j,-} n_{j,-}}{n_{j,+}+n_{j,-}} .
$$

For thermal populations of the components at $\Delta E_{j} / k_{B} T$ $\ll 1, n_{j,+} \approx n_{j,-}$ such that $\widetilde{\chi}_{j}^{\mathrm{eff}} \approx \chi_{j}$. At the lower limit of the temperature range $T \gg T^{\Lambda}$, near-adiabatic conditions are attained when $\tilde{\chi}_{j,-} \ll \tilde{\chi}_{j,+}$ such that $\widetilde{\chi}_{j}^{\text {eff }} \approx \tilde{\chi}_{j,+} / 2$. For nonthermal populations with arbitrary relation between $n_{j,+}$ and $n_{j,-}, \widetilde{\chi}_{j}^{\text {eff }}$ can range from $\tilde{\chi}_{j,+}$ (when only the lower component is populated) to $\tilde{\chi}_{j,-}$ (when only the upper component is populated).

Quite general analytical results can be obtained under the condition that the AC potentials are calculated in the PR approximation. For instance, the AC PR potentials for $\Omega$ $=1 / 2, j=1 / 2$, where the cq term vanishes, read as ${ }^{11}$

$$
V_{1 / 2, m}^{\mathrm{PR}}(R)=-\frac{2 m \mu_{D} q}{3 R^{2}}-\frac{q^{2} \alpha^{\mathrm{PR}}}{2 R^{4}},
$$

where $\alpha^{\mathrm{PR}}$ is the effective $\mathrm{PR}$ polarizability, $\alpha^{\mathrm{PR}}=\alpha$ $+(4 / 27) \mu_{D}^{2} / B$, which is defined through $\alpha, \mu_{D}$ [see Eq. (1)], and the rotational constant $B$ of the diatom. ${ }^{11}$

The capture rate coefficient $\chi_{1 / 2}^{\mathrm{PR}}=\chi_{1 / 2,1 / 2}^{\mathrm{PR}}+\chi_{1 / 2,-1 / 2}^{\mathrm{PR}}$ depends on the temperature through the reduced temperature $\theta^{\mathrm{PR}}=k_{B} T / E_{1 / 2,-1 / 2}^{* \mathrm{PR}}, E_{1 / 2,-1 / 2}^{* \mathrm{PR}}=\mu_{D}^{2} / 18 \alpha^{\mathrm{PR}}$, and its partial contributions read as

$$
\begin{aligned}
& \chi_{1 / 2,1 / 2}^{\mathrm{PR}}\left(\theta^{\mathrm{PR}}\right)=\sqrt{\alpha^{P R} / \alpha}\left(1 / \sqrt{\pi \theta^{\mathrm{PR}}}+1 / 2\right), \\
& \chi_{1 / 2,-1 / 2}^{\mathrm{PR}}\left(\theta^{\mathrm{PR}}\right)=\sqrt{\alpha^{P R} / \alpha}\left((1 / 2) \operatorname{erfc}\left(1 / \sqrt{\theta^{\mathrm{PR}}}\right)\right),
\end{aligned}
$$

where $\operatorname{erfc}(x)$ is the complementary error function, $\operatorname{erfc}(x)$ $=(2 / \pi) \int_{x}^{\infty} \exp \left(-y^{2}\right) d y$. The asymptotic forms of $\chi_{1 / 2}^{\mathrm{PR}}\left(\theta^{\mathrm{PR}}\right)$ are 


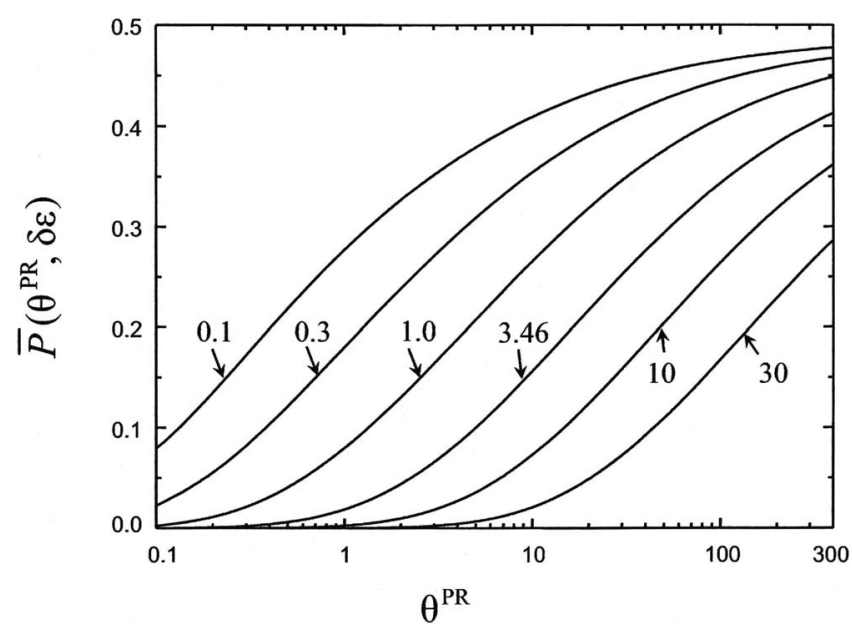

FIG. 6. Mean transition probabilities $\bar{P}\left(\theta^{\mathrm{PR}}, \delta \varepsilon\right)$ expressing $\tilde{\chi}_{1 / 2,+}^{\mathrm{PR}}$ and $\tilde{\chi}_{1 / 2,-}^{\mathrm{PR}}$ through $\chi_{1 / 2,+1 / 2}^{\mathrm{PR}}$ and $\chi_{1 / 2,-1 / 2}^{\mathrm{PR}}$ in Eq. (34). The curves are labeled by the values of $\delta \varepsilon$. The value $\delta \varepsilon=3.46$ refers to the capture of $\mathrm{NO}\left(X^{2} \Pi_{1 / 2}, j\right.$ $=1 / 2)$ by $\mathrm{C}^{+}$.

$$
\chi_{1 / 2}^{\mathrm{PR}}\left(\theta^{\mathrm{PR}}\right)= \begin{cases}\sqrt{\alpha^{\mathrm{PR}} / \pi \alpha \theta^{\mathrm{PR}}}, & \theta^{\mathrm{PR}} \ll 1 \\ \sqrt{\alpha^{\mathrm{PR}} / \alpha}, & \theta^{\mathrm{PR}} \gg 1 .\end{cases}
$$

Note that the high temperature limit $\left(\theta^{\mathrm{PR}} \gg 1\right)$ is $\sqrt{\alpha^{\mathrm{PR}} / \alpha}$ and not unity (that would correspond to the sum of partial contributions of $\chi_{1 / 2,1 / 2}$ and $\left.\chi_{1 / 2,-1 / 2}\right)$ since the effective PR polarizability $\alpha^{\mathrm{PR}}$ differs from $\alpha$.

Beside the trivial scaling factor $\sqrt{\alpha^{\mathrm{PR}} / \alpha}$, the rate coefficients $\tilde{\chi}_{1 / 2,+}^{\mathrm{PR}}$ and $\tilde{\chi}_{1 / 2,-}^{\mathrm{PR}}$ depend on two parameters: the reduced temperature $\theta^{\mathrm{PR}}$ and the reduced $\Lambda$-doubling splitting $\delta \varepsilon=\left(\Delta E_{1 / 2} / E_{1 / 2,-1 / 2}^{* \mathrm{PR}}\right)\left(q \mu \mu_{D} / 6 \hbar^{2}\right)$. We can then write

$$
\begin{aligned}
& \tilde{\chi}_{1 / 2,+}^{\mathrm{PR}}=2(1-\bar{P}) \chi_{1 / 2,+1 / 2}^{\mathrm{PR}}+2 \bar{P} \chi_{1 / 2,-1 / 2}^{\mathrm{PR}}, \\
& \tilde{\chi}_{1 / 2,-}^{\mathrm{PR}}=2 \bar{P} \chi_{1 / 2,+1 / 2}^{\mathrm{PR}}+2(1-\bar{P}) \chi_{1 / 2,-1 / 2}^{\mathrm{PR}} .
\end{aligned}
$$

Here, the mean transition probability $\bar{P}$ has the expected values $\bar{P}=0$ and $\bar{P}=1 / 2$ for the adiabatic and sudden limits, respectively. For the intermediate case, the quantity $\bar{P}$ serves as a means to express the two rate coefficients $\tilde{\chi}_{1 / 2,+}^{\mathrm{PR}}$ and $\tilde{\chi}_{1 / 2,-}^{\mathrm{PR}}$ through the rate coefficients $\chi_{1 / 2,1 / 2}^{\mathrm{PR}}$ and $\chi_{1 / 2,-1 / 2}^{\mathrm{PR}}$ from the analytical Eq. (32) and $\bar{P}=\bar{P}\left(\theta^{\mathrm{PR}}, \delta \varepsilon\right)$. Plots of the function $\bar{P}\left(\theta^{\mathrm{PR}}, \delta \varepsilon\right)$ are shown in Fig. 6 for different values of $\delta \varepsilon$. The value $\delta \varepsilon=3.46$ refers to the capture of $\mathrm{NO}\left(X^{2} \Pi_{1 / 2}, j\right.$ $=1 / 2$ ) by $\mathrm{C}^{+}$such as discussed in Ref. 11 .

\section{RATE COEFFICIENTS FOR $\Lambda$-DOUBLING SPECIFIC CAPTURE IN THE ADIABATIC REGIME}

At $T \approx T^{\Lambda}$ and below, capture occurs adiabatically with respect to transitions between the components of a $\Lambda$-doublet and, therefore, the rate coefficient can be calculated in the framework of the AC approximation with AC potentials calculated by taking the $\Lambda$-doubling splitting into account. In addition, one can use the PR approximation. Therefore, one can employ Eqs. (3)-(6) for numerical calculations of the capture rate coefficients $\tilde{\chi}_{j, m}^{\mathrm{PR}}$. However, a useful analytical result can be also be obtained when one neglects the chargequadrupole and the effective Langevin interactions against the intermediate Stark charge-dipole interaction. Although the leading asymptotic interaction (in the limit $R \rightarrow \infty$ ) is dominated by the charge-quadrupole term proportional to $R^{-3}$, there might exist a broad range of temperatures, within which the $R^{-3}$ interaction term can be neglected in comparison to the $R^{-4}$ term (since the latter contains a large factor which is inversely proportional to $\Delta E_{j}$ ) unless the dipole moment of the diatom is anomaly small.

We, therefore, consider a case where at all separations, the AC PR potentials are well represented by the chargepermanent dipole (cd) interaction in the intermediate Stark regime [Eq. (7)]. Since $\left.\tilde{V}_{j, m}^{\text {cd }}(R)\right|_{m<0}$ are repulsive, the capture rate coefficients $\left.\tilde{\chi}_{j, m}^{\mathrm{cd}}\right|_{m<0} ^{j, m}$ and $\widetilde{\chi}_{j,-}^{\mathrm{cd}}$ vanish. The capture rate coefficients $\tilde{\chi}_{j, m}^{\text {cd }}$ for $m>0$ are then determined by the general expression [Eq. (22)],

$$
\widetilde{\chi}_{j, m}^{\mathrm{cd}}=2 A(T) \int_{0}^{\infty} \frac{J d J}{2 j+1} \exp \left(-\tilde{U}_{j, m}^{\mathrm{cd}, \max }(J) / k_{B} T\right) .
$$

Here, $\tilde{U}_{j, m}^{\mathrm{cd}, \max }(J)$ is the maximum of the effective potential

$$
\tilde{U}_{j, m}^{\mathrm{cd}}(R, J)=\hbar^{2}(J+1 / 2)^{2} / 2 \mu R^{2}+\tilde{V}_{j, m}^{\mathrm{cd}}(R) .
$$

For the cd potential $\widetilde{V}_{j, m}^{\text {cd }}(R)$ from Eq. (7), the centrifugal barrier $\tilde{U}_{j, m}^{\text {cd,max }}(J)$ can be calculated analytically as

$$
\tilde{U}_{j, m}^{\mathrm{cd}, \max }(J)=\frac{\Delta E_{j}}{2}\left(1-\sqrt{1-J^{4} / J_{j, m}^{4}}\right),
$$

where $J_{j, m}=\sqrt{2 \mu a_{j, m} / \hbar^{2}}$. Equation (37) then shows that the upper integration limit in Eq. (35) is actually $J=J_{j, m}$, the largest relative angular momentum for which $\left.\tilde{U}_{j, m}(R, J)\right|_{\Delta E_{j}=0}$ becomes nonattractive.

When the expression for $\tilde{U}_{j, m}^{\mathrm{cd}, \max }(J)$ from Eq. (37) is substituted into Eq. (35) and the integration variable is properly modified, one finds that $\Lambda$-doubling in the rate coefficient $\widetilde{\chi}_{j, m}^{\mathrm{cd}}$ manifests itself by the appearance of an $m$-independent factor $F=F\left(\theta_{j}^{\Lambda}\right)$ in front of the capture rate coefficient $2 \chi_{j, m}^{\mathrm{cd}}, \theta_{j}^{\Lambda}$ being defined by $\theta_{j}^{\Lambda}=k_{B} T / \Delta E_{j}$. This leads to

$$
\begin{aligned}
\tilde{\chi}_{j, m}^{\mathrm{cd}}(T)= & 2 A(T) \int_{0}^{J_{j, m}} \frac{J d J}{2 j+1} \\
& \times \exp \left\{-\frac{\Delta E_{j}}{2 k_{B} T}\left(1-\sqrt{1-J^{4} / J_{j, m}^{4}}\right)\right\} \\
= & 2 \chi_{j, m}^{\mathrm{cd}}(T) \times F\left(\theta_{j}^{\Lambda}\right),
\end{aligned}
$$

where $\chi_{j, m}^{\mathrm{cd}}$ is given by Eq. (25) and the function $F=F\left(\theta_{j}^{\Lambda}\right)$ is defined as

$$
F\left(\theta_{j}^{\Lambda}\right)=\int_{0}^{1} d y \exp \left[-\left(1 / 2 \theta_{j}^{\Lambda}\right)\left(1-\sqrt{1-y^{2}}\right)\right]
$$

It is important to note that this function damps the $T^{-1 / 2}$ divergence of the rate coefficient $\chi_{j, m}^{\mathrm{cd}}(T)$ and has the following asymptotic forms: 


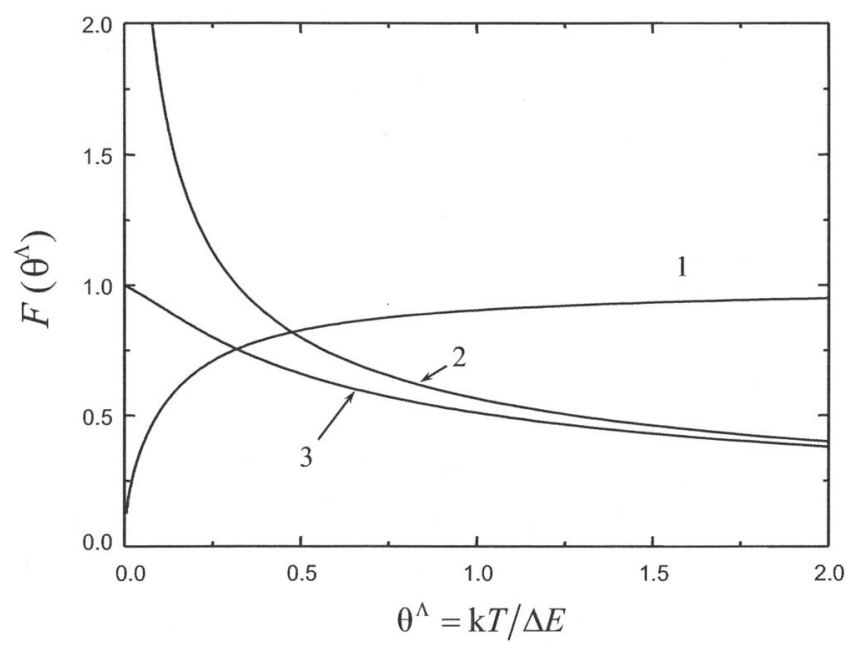

FIG. 7. Damping function $F\left(\theta^{\Lambda}\right)$, Eq. (39) (curve 1), undamped function $\sqrt{\pi \theta^{\Lambda}}$ (curve 2), and its damped counterpart $\Phi\left(\theta^{\Lambda}\right)$, Eq. (45) (curve 3) as functions of $\theta^{\Lambda}=k_{B} T / \Delta E$.

$$
F\left(\theta_{j}^{\Lambda}\right)= \begin{cases}1, & \theta_{j}^{\Lambda} \gg 1 \\ \sqrt{\pi \theta_{j}^{\Lambda}}, & \theta_{j}^{\Lambda} \ll 1 .\end{cases}
$$

The function $F\left(\theta_{j}^{\Lambda}\right)$ and some related functions are shown in Fig. 7.

Since the factor $F$ in Eq. (38) does not depend on $m$, the total rate coefficient for capture of a molecule in the lower $\Lambda$ component of the rotational state $j$ at very low temperatures, $\tilde{\chi}_{j,+}^{\mathrm{cd}}$, is related to the total rate coefficient for the rotational state $j$ with zero $\Lambda$-doublet splitting, $\chi_{j}$, in the following simple way:

$$
\widetilde{\chi}_{j,+}^{\mathrm{cd}}(T)=2 \chi_{j}^{\mathrm{cd}}(T) F\left(\Delta E_{j} / k_{B} T\right)
$$

Equation (41) possesses the following limits for $T \gg \Delta E_{j} / k_{B}$ and $T \ll \Delta E_{j} / k_{B}$ :

$$
\tilde{\chi}_{j,+}^{\mathrm{cd}}(T)=\left\{\begin{array}{cc}
2 \chi_{j}^{\mathrm{cd}}(T) & \text { for } T \gg \Delta E_{j} / k_{B} \\
2 \chi_{j}^{\mathrm{cd}}(T) \sqrt{\pi \theta_{j}^{\Lambda}} & \text { for } T \ll \Delta E_{j} / k_{B} .
\end{array}\right.
$$

This expression shows that for $T \gg \Delta E_{j} / k_{B}$ the rate $\tilde{\chi}_{j,+}^{\mathrm{cd}}(T)$ is expressed through the adiabatic limit of the rate constant $\chi_{j}^{\mathrm{cd}}(T)$. In the opposite case, for $T \ll \Delta E_{j} / k_{B}$, the rate coefficient $\widetilde{\chi}_{j,+}^{\mathrm{cd}}(T)=2 \chi_{j}^{\mathrm{cd}}(T) \sqrt{\pi k_{B} T / \Delta E_{j}}$ coincides with the scaled effective Langevin capture rate coefficient

$$
\left.\widetilde{\chi}_{j,+}^{\mathrm{cd}}(T)\right|_{k_{B} T \ll \Delta E_{j}^{\Lambda}} \equiv \widetilde{\chi}_{j,+}^{\mathrm{cd}-\mathrm{L}}=\sqrt{\tilde{\alpha} / \alpha},
$$

where the factor $\tilde{\alpha}$ is expressed via the effective partial polarizabilities $\tilde{\alpha}_{j, m}$ and ultimately through the dipole moment and the $\Lambda$-doubling splitting,

$$
\begin{aligned}
& \sqrt{\widetilde{\alpha}}=\frac{1}{2 j+1} \sum_{m} \sqrt{\widetilde{\alpha}_{j, m}}, \\
& \tilde{\alpha}_{j, m}=2 \mu_{D}^{2} m^{2} \Omega^{2} / j^{2}(j+1)^{2} \Delta E_{j} .
\end{aligned}
$$

Of course, the Langevin capture rate coefficient $\widetilde{\chi}_{j,+}^{\mathrm{cd}-\mathrm{L}}$ coincides with the total capture rate coefficient expressed through the partial capture rate coefficient $\widetilde{\chi}_{j, m}^{\mathrm{cd}-\mathrm{L}}, \widetilde{\chi}_{j,+}^{\mathrm{cd}-\mathrm{L}}=\Sigma_{m} \widetilde{\chi}_{j, m}^{\mathrm{cd}-\mathrm{L}}$, which, in turn, are calculated for the effective Langevin attractive potentials $\tilde{V}_{j, m}^{\mathrm{cd}-\mathrm{L}}(R)=\left.\tilde{V}_{j, m}^{\mathrm{cd}}(R)\right|_{\Delta E_{j} \gg a_{j, m / R^{2}}}=-q^{2} \widetilde{\alpha}_{j, m} /$ $2 R^{4}$.

Since within the above treatment, the relative motion of the partners is described classically even in the low temperatures, $\theta_{j}^{\Lambda} \ll 1$, one has to ensure that the contribution to the integral comes from $J$-values that are notably larger than unity. Indeed, inspection of the integrand in Eq. (39)) for $\theta_{j}^{\Lambda} \ll 1$ shows that this condition is fulfilled provided that $\left(\theta_{j}^{\Lambda}\right)^{1 / 4} J_{j, m} \gg 1$. This inequality can be reformulated as the condition that the temperature $T$ is substantially higher than the characteristic ultralow temperature $T_{j, m}^{\mathrm{ULT}}$ for each individual capture channel given by $T_{j, m}^{\mathrm{ULT}}=\hbar^{4} / \mu^{2} q^{2} \tilde{\alpha}_{j, m}$. The latter condition guaranties the good performance of the classical approximation for calculating the capture rate coefficient with the potentials of Eq. (36). Assuming that the condition $T \gg T_{j, m}^{\mathrm{ULT}}$ is fulfilled for all capture channels, we see that the ratio $\widetilde{\chi}_{j,+}^{\mathrm{cd}} / \widetilde{\chi}_{j}^{\mathrm{cd}-\mathrm{L}}$ depends only on a single variable $\theta_{j}^{\Lambda}$ $=k_{B} T / \Delta E_{j}$, and can be represented as a universal function

$$
\begin{aligned}
& \widetilde{\chi}_{j,+}^{\mathrm{cd}} / \widetilde{\chi}_{j,+}^{\mathrm{cd}-\mathrm{L}}=\Phi\left(\theta_{j}^{\Lambda}\right), \\
& \Phi\left(\theta_{j}^{\Lambda}\right)=\frac{1}{\sqrt{\pi \theta_{j}^{\Lambda}}} F\left(\theta_{j}^{\Lambda}\right) .
\end{aligned}
$$

The rate coefficient $\widetilde{\chi}_{j,+}^{\mathrm{cd}-\mathrm{L}}$ is expected to provide the dominant contribution to the accurate rate coefficient $\tilde{\chi}_{j,+}^{\mathrm{PR}}$ in the limit $k_{B} T \ll \Delta E_{j}$. In this limit, the ratio $\tilde{\chi}_{j,+}^{\mathrm{PR}} / \widetilde{\chi}_{j,+}^{\mathrm{cd}-\mathrm{L}}$ differs from unity by a term of the order of $\alpha^{\mathrm{PR} /} \tilde{\alpha}$ which normally is very small [with $\widetilde{\alpha}$ from Eq. (44) and $\alpha^{\mathrm{PR}}$ from Eq. (31)]. The difference between $\tilde{\chi}_{j,+}^{\mathrm{PR}}$ and $\tilde{\chi}_{j,+}^{\mathrm{cd}}$ is due to the neglect of the effective Langevin interaction, the term with the polarizability $\alpha^{\mathrm{PR}}$, and the charge-quadrupole interaction. The effect of the former can be easily accounted for while the latter cannot be considered in a simple way, and one has to resort to a general treatment for the potential of the form of Eq. (8) (see Ref. 13). However, due to large coefficients of the $R^{-4}$ term, the effect of the $R^{-3}$ term will show up only at extremely low temperatures where the hyperfine structure cannot be ignored.

On the basis of the present discussion, one can therefore recommend the following general expression for the capture rate coefficients across the whole temperature range (provided that the relative motion of the partners is classical and hyperfine interaction is neglected):

$$
\tilde{\chi}_{j,+}^{\mathrm{PR}}(T) \approx \chi_{j}^{\mathrm{PR}}(T) F\left(\theta_{j}^{\Lambda}\right) .
$$

The satisfactory performance of this formula across a wide temperature range, from $T \ll T^{\Lambda}$ to $T \gg T^{\Lambda}$, is guaranteed by the fact that, when damping is important, the capture is dominated by the cd interaction and, when the PR Langevin correction becomes noticeable, the damping is not important.

\section{DISCUSSION AND CONCLUSION}

The $\Lambda$-doubling specificity of capture rate coefficients is the entrance-channel counterpart of the $\Lambda$-doubling propensity in the formation of diatomic products in the exit channels of decomposing statistical complexes. The latter process has been thoroughly studied by the statistical-closed-coupled $\operatorname{method}^{15,16}$ as applied to the calculation of state-resolved 
cross sections and rate coefficients for the formation of $\mathrm{OH}\left({ }^{2} \Pi\right)$ and $\mathrm{OD}\left({ }^{2} \Pi\right)$ (see Refs. 17 and 18, respectively). The method employed in these papers takes into account all electronic and spin-orbit couplings in the exit channels, and the propensity toward the population of the $\Lambda$-doubling components, presumably created in the complex, was found to survive "the myriad of crossings with the repulsive potentials which all coalesce as the fragments separate." ${ }^{\prime 18}$ The interpretation of the origin of the propensity suggested in Refs. 38 and 39 (and modified later ${ }^{17}$ ) are based on the consideration of the symmetry of the orbital of the bond to be broken with respect to the plane of rotation of the free fragment. These considerations adopt a picture, which neglects the mixing of the spatial components of the electronic functions of different symmetry under the action of spin-orbit interaction. ${ }^{40}$ Therefore, they are applicable to molecules with Hund's coupling case $b$, such as this is approximately the case for $\mathrm{OH}\left(X^{2} \Pi\right)$, but they do not apply to Hund's coupling case $a$ which is relevant for $\mathrm{NO}\left(X^{2} \Pi\right)$. We note in passing that the lowering of the asymmetry of the charge distribution in a rotating diatomic molecule, when the a diatom passes from the coupling case $b$ to $a$ is well-documented phenomenon (see the "Application 15" of Ref. 31 for a comparison of $\mathrm{OH}$ with NO).

Contrary to the full statistical-closed-coupled method, the approach in the present work is considerably less demanding since it takes into account simplifications that arise in the low-energy regime of the capture of the molecule in the case of Hund's coupling case $a$. These features allow one to consider the long-range interaction for a single electronic PES, neglect the coupling between the AC states that correlate with different rotational quantum numbers $j$, and treat the relative motion classically. The only coupling included in the treatment is the nonadiabatic interaction between $\mathrm{AC}$ states that arise from two $\Lambda$-components belonging to the same $j$, and this coupling is described in the common trajectory approximation. It is important that when the condition of the common trajectory approximation $\left(\Delta E_{j} \ll k_{B} T\right)$ is violated with decreasing temperature, the nonadiabatic interaction can already be ignored, and different trajectories can be assigned to different adiabatic potentials. Quantum effects in the adiabatic relative motion of partners are expected to show up at even lower temperatures.

By reversibility arguments, the capture probability in the entrance channels can be rewritten as a decay probability of the complex into the respective exit channel $j, m$ at a given total energy and total angular momentum. Here, the decay probability is the probability of leaving the potential well across a certain effective AC potential multiplied with the probability of adiabatic survival or nonadiabatic transition on the way to the final state $j, \varepsilon$. If one neglects the small energy correction $\Delta E_{j}$, the $\Lambda$-doubling propensity within two states, $j, \varepsilon$ and $j, \varepsilon^{\prime}$, in an exit channel $j, m$ is governed only by the nonadiabatic transition probability; the propensity disappears when the final translational energy is large enough and the transition probability $P_{j, m}(E)$ reaches its sudden limit, $P_{j, m}(E)=P_{j, m}^{\text {sudd }}(E)=1 / 2$. We thus see that, for two final states $\varepsilon= \pm 1$, the $\Lambda$-doubling propensity is created at very large separations as the result of the dynamic nonadiabatic cou- pling between the AC states converging to different $\Lambda$-doubling components. This illustrates the difference in the mechanisms of the $\Lambda$-doubling propensity for the two different Hund coupling cases and for two different regimes of the final translational energy.

Returning to the $\Lambda$-doubling specificity in state-resolved capture rate coefficients, we reiterate two basic features that allowed us to draw a general conclusion. First, the nonadiabatic transitions between $\Lambda$-doubling AC states occurs at collision energies $E$ which are substantially larger than the $\Lambda$-doubling splitting $\Delta E_{j}$ in a free molecule. This is due to the magnitude of the parameter $\mu \mu_{D} m \Omega / \hbar^{2} j(j+1)$ which normally is quite large (if the dipole moment is not abnormally small and $j$ is not too large) and which ensures a relatively large value of the Massey parameter even under the condition $\Delta E_{j} \ll E$. The same condition permits the common-trajectory description of the nonadiabatic dynamics in the region of an intermediate Stark effect for the chargedipole interaction, and it ensures near-adiabatic capture in the region of potential barriers if these are determined by an interplay between linear Stark effect for cd interaction and other terms of the potential. With a decrease of $E$, the common-trajectory description becomes progressively less adequate but the transition probability becomes so low that one can neglect it altogether and treat the capture as a completely adiabatic event. Second, in the regime of adiabatic capture, an adequate description of the interaction is provided by the perturbed rotor potentials with an intermediate Stark effect for the charge-dipole term, other terms playing a minor role. The applicability of the PR approximation for the determination of potential barriers is guaranteed by the fact that $\Lambda$-doubling splitting $\Delta E_{j}$ is much smaller than the energy of the rotational transition. At lower temperatures, capture occurs in the regime of quadratic Stark effect exceeding other terms. The combination of these results allowed us to suggest an approximate expression for the rate coefficients which describes nonadiabatic dynamics over the wide temperature ranges and corresponds to capture in $\mathrm{AC}$ potentials in an intermediate Stark effect regime.

\section{ACKNOWLEDGMENTS}

Financial support of this work by EU Human Potential Program MCRTN 512302 "The Molecular Universe" is gratefully acknowledged.

\section{APPENDIX: GLOSSARY OF ABBREVIATIONS USED IN THIS PAPER}

AC stands for adiabatic channel, PR stands for perturbed rotor, cd stands for charge-permanent dipole, cid stands for charge-induced dipole, cq stands for charge-quadrupole, and $L$ stands for Langevin.

(ii) $\chi_{j, m}^{\mathrm{AC}}, \chi_{j}^{\mathrm{AC}}$ are the reduced partial $(j, m$-specific $)$ and total $(j$-specific) AC capture rate coefficients calculated with the $\Lambda$-doubling effect ignored (i.e., corresponding to the sudden transition between the $\Lambda$ components). With $\mathrm{AC}$ replaced by $\mathrm{PR}$, the rate coefficients $\chi_{j, m}^{\mathrm{PR}}, \chi_{j}^{\mathrm{PR}}$ corresponds to AC PR poten- 
tials. With AC replaced by cd, the rate coefficients $\chi_{j, m}^{\mathrm{cd}}, \chi_{j}^{\mathrm{cd}}$ correspond to the cd interaction in the linear Stark effect regime.

(iii) $\chi_{j, m}^{\text {surv/trans }}$ are the reduced auxiliary capture rate coefficients corresponding to survival on a $j, m$ AC potential and transition between $j, m \rightarrow j,-m$ AC potentials for the motion across the nonadiabaticity region.

(iv) $\tilde{\chi}_{j, m}, \tilde{\chi}_{j, \varepsilon}$ are the reduced partial $(j, m$-specific) and total $(j, \varepsilon$-specific) rate coefficients for the capture in the linear Stark regime with account taken for nonadiabatic transitions between AC potentials.

(v) $\quad \tilde{\chi}_{j, m}^{\mathrm{PR}}, \tilde{\chi}_{j, \varepsilon}^{\mathrm{PR}}$ are reduced partial $(j, m$-specific $)$ and total $(j, \varepsilon$-specific) AC PR capture rate coefficients calculated with $\Lambda$-doubling effect taken into account. With PR replaced by cd, the rate coefficients $\tilde{\chi}_{j, m}^{\mathrm{cd}}, \tilde{\chi}_{j, \varepsilon}^{\mathrm{cd}}$ correspond to the cd interaction in the intermediate Stark effect regime. With PR replaced by cd-L, the rate coefficients $\widetilde{\chi}_{j, m}^{\mathrm{cd}-\mathrm{L}}, \widetilde{\chi}_{j, \varepsilon}^{\mathrm{cd}-\mathrm{L}}$ correspond to the cd interaction in the quadratic Stark effect regime where the AC PR potentials are represented by an effective Langevintype (L) terms.

${ }^{1}$ A. G. G. M. Tielens, The Physics and Chemistry of the Interstellar Medium (Cambridge University Press, Cambridge, 2005).

${ }^{2}$ E. Herbst, J. Phys. Chem. A 109, 4017 (2005).

${ }^{3}$ E. Herbst, J. Phys.: Conf. Ser. 4, 17 (2005).

${ }^{4}$ I. W. M. Smith, A. M. Sage, N. M. Donahue, E. Herbst, and D. Quan, Faraday Discuss. 133, 137 (2006).

${ }^{5}$ E. Herbst and C. M. Leung, Astrophys. J. 310, 378 (1986).

${ }^{6}$ J. Troe, J. Chem. Phys. 87, 2773 (1987); 105, 6249 (1996).

${ }^{7}$ A. I. Maergoiz, E. E. Nikitin, J. Troe, and V. G. Ushakov, J. Chem. Phys. 105, 6263 (1996).

${ }^{8}$ D. C. Clary, Annu. Rev. Phys. Chem. 41, 61 (1990).

${ }^{9}$ A. G. Wickham, T. S. Stoecklin, and D. C. Clary, J. Chem. Phys. 96, 1053 (1992).

${ }^{10}$ D. C. Clary, T. S. Stoecklin, and A. G. Wickham, J. Chem. Soc., Faraday Trans. 89, 2185 (1993).

${ }^{11}$ E. I. Dashevskaya, I. Litvin, E. E. Nikitin, and J. Troe, Phys. Chem. Chem. Phys. 9, 1559 (2007).

${ }^{12}$ E. I. Dashevskaya, A. I. Maergoiz, I. Litvin, E. E. Nikitin, and J. Troe, J. Chem. Phys. 118, 7313 (2003).
${ }^{13}$ E. I. Dashevskaya, I. Litvin, E. E. Nikitin, and J. Troe, J. Chem. Phys. 120, 9989 (2004).

${ }^{14}$ E. E. Nikitin and J. Troe, Phys. Chem. Chem. Phys. 7, 1540 (2005).

${ }^{15}$ E. J. Rackham, F. Huarte-Larranaga, and D. E. Manolopoulos, Chem. Phys. Lett. 343, 356 (2001).

${ }^{16}$ E. J. Rackham, T. Gonzalez-Lezaña, and D. E. Manolopoulos, J. Chem. Phys. 119, 12895 (2003).

${ }^{17}$ M. H. Alexander, E. J. Rackham, and D. E. Manolopoulos, J. Chem. Phys. 121, 5221 (2004).

${ }^{18}$ S. Atahan, M. H. Alexander, and E. J. Rackham, J. Chem. Phys. 123, 204306 (2005).

${ }^{19}$ M. Quack and J. Troe, Ber. Bunsenges. Phys. Chem. 79, 170 (1975).

${ }^{20}$ K. Takayanagi, J. Phys. Soc. Jpn. 45, 976 (1978).

${ }^{21}$ K. Sakimoto and K. Takayanagi, J. Phys. Soc. Jpn. 48, 2076 (1980).

${ }^{22}$ D. R. Bates, Proc. R. Soc. London, Ser. A 384, 289 (1982).

${ }^{23}$ W. L. Morgan and D. R. Bates, Astrophys. J. 314, 817 (1987).

${ }^{24}$ D. C. Clary, Mol. Phys. 54, 605 (1985).

${ }^{25}$ J. Troe, Ber. Bunsenges. Phys. Chem. 99, 341 (1995).

${ }^{26}$ M. Auzinsh, E. I. Dashevskaya, I. Litvin, E. E. Nikitin, and J. Troe (in preparation)

${ }^{27}$ J. J. van Leuken, J. Bulthuis, S. Stolte, and J. G. Snijders, Chem. Phys. Lett. 260, 595 (1996).

${ }^{28}$ A. Gijsbertsen, H. Linnartz, G. Rus, A. E. Wiskerke, S. Stolte, D. W. Chandler, and J. Kłos, J. Chem. Phys. 123, 224305 (2005).

${ }^{29}$ S. Y. T. van de Meerakker, P. H. M. Smeets, N. Vanhaecke, R. T. Jongma, and G. Meijer, Phys. Rev. Lett. 94, 023004 (2005).

${ }^{30}$ J. J. Gilijamse, S. Hoekstra, S. Y. T. van de Meerakker, G. C. Groenenboom, and G. Meijer, Science 313, 1617 (2006).

${ }^{31}$ R. N. Zare, Angular Momentum (Wiley, New York, 1988).

${ }^{32}$ M. H. Alexander, J. Chem. Phys. 76, 5974 (1982).

${ }^{33}$ T. Orlikowski and M. H. Alexander, J. Chem. Phys. 79, 6006 (1983).

${ }^{34}$ M. H. Alexander, Faraday Discuss. 113, 437 (1999).

${ }^{35}$ S. C. Smith and J. Troe, J. Chem. Phys. 97, 5451 (1992).

${ }^{36}$ J. D. Graybeal, Molecular Spectroscopy (McGraw-Hill, New York, 1988).

${ }^{37}$ E. E. Nikitin and S. Ya. Umanskii, Theory of Slow Atomic Collisions (Springer, Berlin, 1984).

${ }^{38}$ J. E. Butler, G. M. Jursich, I. A. Watson, and J. R. Wiesenfeld, J. Chem. Phys. 84, 5365 (1986).

${ }^{39}$ M. J. Bronikowski and R. N. Zare, Chem. Phys. Lett. 166, 5 (1990).

${ }^{40}$ M. H. Alexander, P. Andresen, R. Bacis, R. Bersohn, F. J. Comes, P. J. Dagdigian, R. N. Dixon, R. W. Field, G. W. Flynn, K.-H. Gericke, E. R. Grant, B. J. Howard, J. R. Huber, D. S. King, J. L. Kinsey, K. Kleinermanns, K. Kuchitsu, A. C. Luntz, A. J. McCaffery, B. Pouilly, H. Reisler, S. Rosenwaks, E. W. Rothe, M. Shapiro, J. P. Simons, R. Vasudev, J. R. Wiesenfeld, C. Wittig, and R. N. Zare, J. Chem. Phys. 89, 1749 (1988). 